\title{
Acustimulation Therapy
}

National Cancer Institute

\section{Source}

National Cancer Institute. Acustimulation Therapy. NCI Thesaurus. Code C15761.

Use of a low intensity electrical current to provide mild stimulation of the body's acupuncture points in order to control symptoms such as nausea and vomiting. 